\title{
Application of ultrasound-guided external jugular vein puncture in intensive care unit (ICU) patients with severe sepsis: a randomised trial
}

\author{
Sha Luo ${ }^{1 \#}$, Yunna Xue ${ }^{2 \#}$, Sheng Tang ${ }^{3}$, Shujuan $\mathrm{Hou}^{3}$, Minjie Pei ${ }^{3}$ \\ ${ }^{1}$ Department of Nephrology, Chinese PLA General Hospital, Chinese PLA Institute of Nephrology, State Key Laboratory of Kidney Diseases, \\ National Clinical Research Center for Kidney Diseases, Beijing Key Laboratory of Kidney Diseases, Beijing, China; ${ }^{2}$ Department of Neurological \\ Intensive Care Unit, the First Medical Centre, Chinese PLA General Hospital, Beijing, China; ${ }^{3}$ Department of Critical Care Medicine, the First \\ Medical Centre, Chinese PLA General Hospital, Beijing, China \\ Contributions: (I) Conception and design: S Luo, Y Xue, S Tang; (II) Administrative support: Y Xue, S Hou; (III) Provision of study materials or \\ patients: None; (IV) Collection and assembly of data: S Luo, Y Xue, S Hou; (V) Data analysis and interpretation: S Luo, Y Xue, S Tang; (VI) \\ Manuscript writing: All authors; (VII) Final approval of manuscript: All authors. \\ "These authors contributed equally to this work. \\ Correspondence to: Sheng Tang. Department of Critical Care Medicine, the First Medical Centre, Chinese PLA General Hospital, 28 Fuxing Road, \\ Beijing 100853, China. Email: ts00307@163.com.
}

Background: Sepsis is one of the most common critical illnesses in intensive care unit (ICU) clinical practice. Intravenous infusion technology is an important method for life support. The commonly used deep vein indwelling is expensive, and the incidence of infection due to long-term placement is high. Ultrasound technology can guide clinical puncture operations in real time and greatly improve the success rate of puncture. In the present study, we aimed to explore the effect of ultrasound-guided external jugular vein puncture and catheterization in patients with sepsis.

Methods: From December 2018 to December 2019, a total of 61 patients with sepsis from the ICU or neurology care unit (NCU) were included in the present study and were randomly divided into the experimental group (n=30) and control group (n=31). Ultrasound-guided indwelling cannula needle was used in the experimental group, and blind indwelling cannula needle was used in the control group. The success rate of puncture at first operation, indwelling complications (e.g., bleeding, redness, infection, poor infusion), and operation time of the 2 methods were compared.

Results: A comparison of the patients in the control group with the experimental group indicated no significant differences in age, body mass index, sex, temperature, mean aortic pressure, sequential organ failure score, need for vasopressors, central venous pressure, leucocytes, hemoglobin, platelets, albumin, and mechanical ventilation (all $\mathrm{P}>0.05$ ). The duration of ICU or NCU stay was 5 days in the experimental group compared with 7 days in the control group $(\mathrm{P}=0.009)$. Compared with the control group, the experimental group had shorter successful puncture time (4.0 vs. $6.0 \mathrm{~min}, \mathrm{P}<0.001)$, higher first puncture success rate $(93 \%$ vs. $71 \%, \mathrm{P}=0.023)$, and a lower incidence of complications $(12.5 \%$ vs. $45.0 \%, \mathrm{P}=0.001)$.

Conclusions: For patients with sepsis in ICU, ultrasound-guided puncture is superior to blind manual puncture.

Keywords: Ultrasound; external jugular vein puncture; intensive care unit; sepsis

Submitted Oct 16, 2020. Accepted for publication Jan 18, 2021.

doi: 10.21037/apm-20-2500

View this article at: http://dx.doi.org/10.21037/apm-20-2500 


\section{Introduction}

Sepsis is one of critical illnesses seen in intensive care unit (ICU) clinical practice, and is often induced by factors, such as severe trauma, burns, major surgery, and infection. The condition of patients with sepsis deteriorates rapidly and even results in septic shock and multiple organ dysfunction. Although there are good diagnostic and treatment techniques and monitoring measures, the incidence and mortality of sepsis are still high. Doctors and investigators are faced with many serious problems. Intravenous infusion technology is one of the important measures to maintain the channel of medical treatment for patients, which directly determines the timeliness and effectiveness of treatment. The clinically commonly used deep vein indwelling is expensive, and the incidence of long-term placement infection is high. Therefore, choosing an infusion pathway that can reduce repeated punctures, increase rapid patency of the drug solution, reduce venous catheterization complications, and prolong the indwelling time is a critical clinical issue that needs to be considered. In recent years, with the promotion of ultrasound, ultrasoundguided peripheral venipuncture has become a new alternative. Ultrasound technology can locate the blood vessel and observe the inner diameter of the blood vessel, thickness of the wall, and depth from the body surface (1), which can guide the clinical puncture operation in real time and greatly improve the success rate of puncture. It plays an important auxiliary role in the management and treatment of critically ill patients, and is a new auxiliary method to improve the success rate of the first puncture (2). There are few studies on the feasibility of external jugular vein puncture and operation under ultrasound guidance, especially for patients with sepsis. Deep vein puncture in patients with sepsis are often at high risk of infection and operation failure. Therefore, the aim of the present study was to explore the effect of ultrasound-guided external jugular vein puncture and catheterization in patients with sepsis. We present the following article in accordance with the CONSORT reporting checklist (available at http:// dx.doi.org/10.21037/apm-20-2500).

\section{Methods}

\section{Research participants}

A total of 61 patients with sepsis treated in the ICU or neurology care unit in the General Hospital of the People's Liberation Army from December 2018 to December 2019 were enrolled, including 34 males and 27 females, according to the inclusion and exclusion criteria. The inclusion criteria were as follows: (I) age $>18$ years; (II) hospital stay $>48 \mathrm{~h}$; and (III) case data with complete information. Exclusion criteria were as follows: (I) patients with abnormal coagulation function; (II) those who had just had neck surgery; and (III) neck skin ulcerations. The definition of sepsis was based on the new definition and new diagnostic standard sepsis-3 (3). Patients with sepsis who had an infection and sequential organ failure score (SOFA) score $>2$ were included as the research participants. The present study was approved by the Ethics Committee of the First Medical Center of the PLA General Hospital (approval number: 20200328), and written informed consent was provided by the patient or family member. All procedures performed in this study involving human participants were in accordance with the Declaration of Helsinki (as revised in 2013).

\section{Research grouping}

Sixty-one patients were divided into the experimental group and control group by random number table method.

\section{Research methods}

A Braun safety venous indwelling needle (18G, Melsungen, Germany) was used for the puncture, and the operations were performed by senior nurses who had been trained in external jugular vein puncture specifications and experienced in puncture. In the experimental group $(n=30)$, the Philips PureWave ultrasound system was used, which is equipped with a high-frequency probe (L12-3), and adopts ultrasound guidance to locate the external jugular vein puncture catheter. Traditional blind puncture was used in the control group $(n=31)$ to compare the operation time and success rate of 1 puncture between the 2 groups. Complications included liquid extravasation, subcutaneous hematoma, and phlebitis. In practice, only doctors with sophisticated experience were permitted to do this.

\section{Experimental group}

The patient was placed in the supine position with a thin pillow under the shoulders and the head tilted to the opposite side, with 1 side of the neck fully exposed. The puncture site was routinely disinfected with iodophor, and the ultrasound probe was coated with a coupling agent and covered with a disposable sterile probe protective cover. 


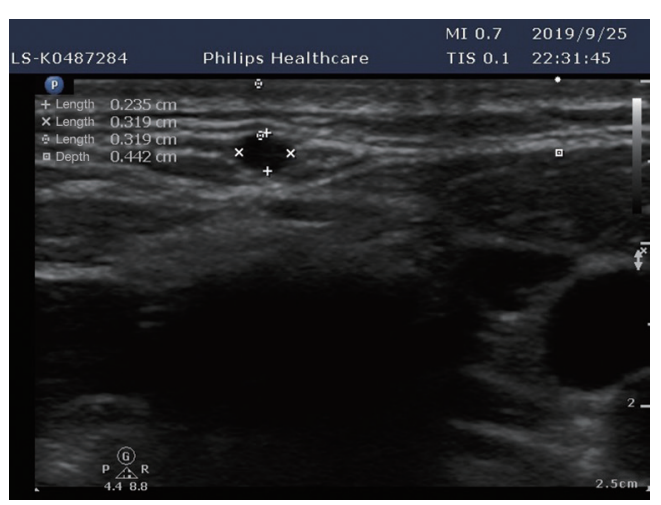

Figure 1 Detection of external jugular vein.

A hand-held ultrasound probe was used to evaluate the position of the patient's external jugular vein. The blood vessel was selected, and the image was placed in the middle of the short-axis plane. We determined the position, shape, and depth of the external jugular vein (Figure 1), selected the puncture point, and the direction of needle insertion. The 18\#-22\# trocar was held in the other hand from the midpoint of the probe and the needle was inserted at an angle of $15-30^{\circ} 1 \mathrm{~cm}$ upwards. Once the blood returned into the syringe, the needle core was withdrawn while pushing the cannula, and the needle core was then pulled out. The trocar was fixed and the tube was successfully inserted. The brief operation process is shown in Figure 2. If the puncture was unsuccessful, the tube was removed and the gauze pressed against the puncture site.

\section{Control group}

The patient was placed in the supine position with a thin pillow under the shoulders. The point where the external jugular vein expanded well or stimulated the patient to cough to make the vein well exposed was chosen. The patient's head was tilted to the opposite side. Routine disinfection was performed. The middle point between the mandibular angle and the upper edge of the clavicle was selected as the puncture point. For the puncture point, the proximal end of the blood vessel was pressed with the left hand under direct vision, and the intravenous indwelling needle was used with the right hand at an angle of $15-20^{\circ}$; the skin was tightened. If the blood returned to the syringe, the needle core was withdrawn before sending the cannula into vessels. If the puncture was successful, the applicator was fixed. If the puncture failed, the tube was removed and the gauze was pressed against the puncture site to prevent bleeding.

\section{Evaluation}

The length of time required for successful puncture in the 2 groups was recorded, and we compared the success rate of first puncture and the incidence of complications (e.g., liquid extravasation, subcutaneous hematoma, phlebitis) within 7 days after catheter placement.

\section{Statistical analysis}

SPSS 20.0 (IBM, Armonk, NY, USA) statistical software was used for the data analysis. Continuous variables were expressed as mean \pm standard deviation, and compared by 2 independent samples $t$-tests. Categorical variables were expressed by rate or composition ratio, and $\chi^{2}$-test was used. $\mathrm{P}<0.05$ indicated statistical significance.

\section{Results}

\section{Baseline characteristics}

There were 30 cases in the experimental group. Ten cases had sepsis caused by multiple injuries, 6 had abdominal infection, 5 had severe pancreatitis, 7 had urinary tract infection, 1 had right kidney tumor, and 1 was parturient. There were 31 cases in the control group. Nine cases had multiple trauma sepsis, 8 had abdominal infection, 7 had severe pancreatitis, 5 had postoperative sepsis of gastric cancer, and 2 had aortic dissection. The 61 patients had a median age of 56 years; the median age of the experimental group was 57 years and that of the control group was 55 years. There were no statistically significant differences in the comparison of the 2 groups of patients with regard to age, sex ratio, body mass index, body temperature, mean arterial pressure, SOFA score, use of blood pressure drugs, center venous pressure, white blood cells, hemoglobin, neutrophils, platelets, serum albumin, and mechanical ventilation (all $\mathrm{P}>0.05$ ). The time spent in ICU in the experimental group was significantly shorter ( 5 vs. 7 days, $\mathrm{P}=0.009$ ), and the difference was statistically significant (Table 1).

\section{Comparison of puncture operation between the 2 groups}

There were 22 successful 1-time punctures in the control group with a success rate of $71 \%$. In the experimental group, there were 28 successful 1-time punctures with a success rate of $93 \%$. The difference between the 2 groups was statistically significant $(\mathrm{P}=0.023)$. The distance from 

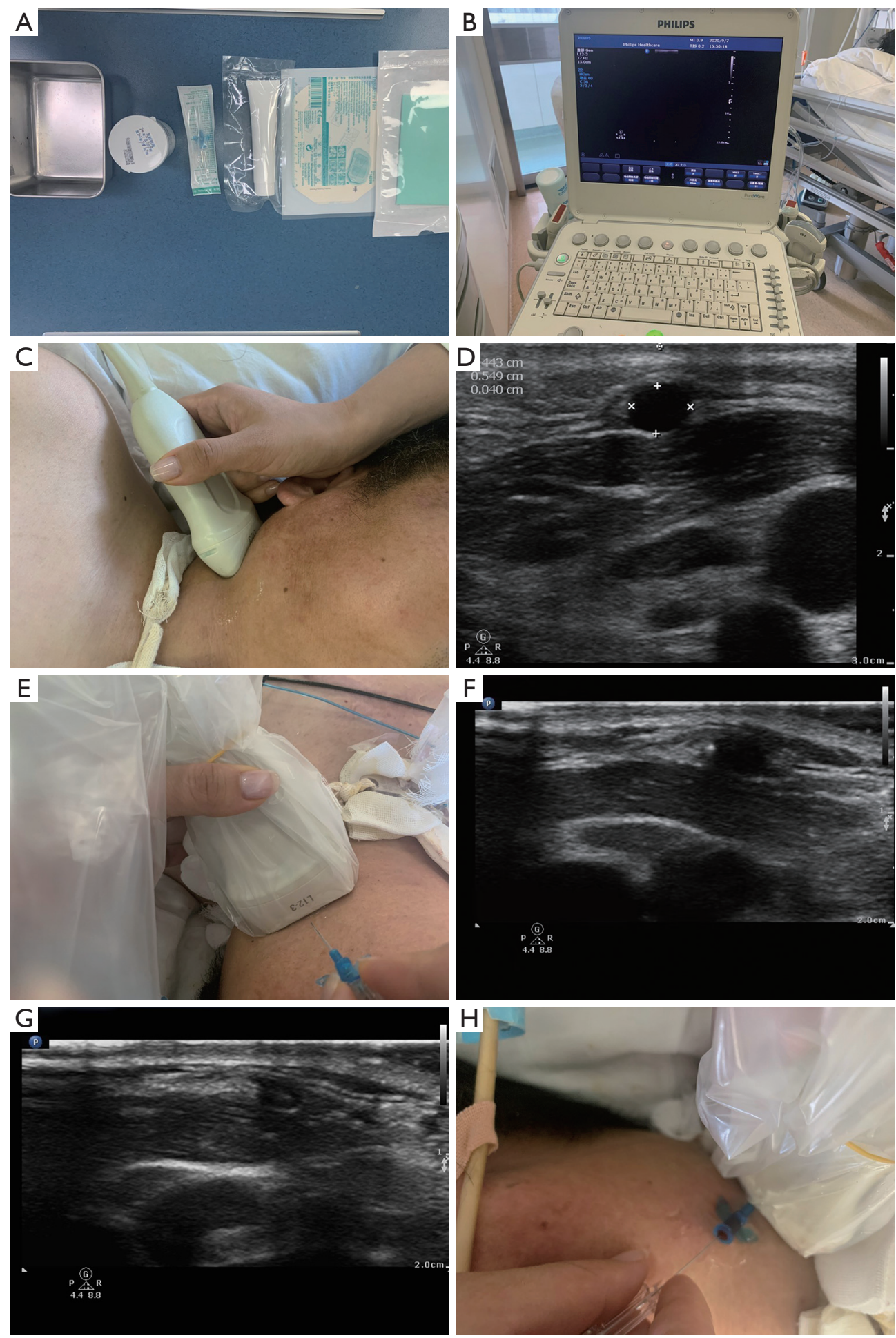

Figure 2 Brief operation process and schematic diagram. (A) Preparation for operation; (B) ultrasound has been prepared; (C) determining the position of the puncture point; (D) determining the depth and diameter of the vein; (E) aseptic hood protects the probe, smears coupling agent, and inserts the needle at an angle of $15-20^{\circ}$; (F) hyperechoic bright spot appears on the tube wall (puncture needle); (G) hyperechoic bright spot appears on the lumen (puncture needle); (H) the needle is successful placed in vein. 
Table 1 Comparison of general information of the 2 groups of patients

\begin{tabular}{|c|c|c|c|c|}
\hline Variables & Total & Experimental group & Control group & $P$ value \\
\hline Age (years) & 56 [44-71] & 57 [46-73] & 55 [43-69] & 0.453 \\
\hline Male, n (\%) & $34(55.7)$ & $19(63.3)$ & $15(48.4)$ & 0.240 \\
\hline BMI $\left(\mathrm{kg} / \mathrm{m}^{2}\right)$ & $23.9 \pm 4.3$ & $24.3 \pm 5.2$ & $23.5 \pm 3.2$ & 0.428 \\
\hline MAP $(\mathrm{mmHg})$ & $77 \pm 11$ & $78 \pm 12$ & $76 \pm 10$ & 0.619 \\
\hline SOFA score & $6.0(4.0-8.0)$ & $6.0(5.0-8.0)$ & $6.0(4.0-8.0)$ & 0.816 \\
\hline Vasoactive drugs, n (\%) & $19(31.1)$ & $10(33.3)$ & $9(29.0)$ & 0.717 \\
\hline CVP $\left(\mathrm{cmH}_{2} \mathrm{O}\right)$ & $8.0(5.0-10.0)$ & $8.0(6.0-10.0)$ & $9.0(5.0-10.0)$ & 0.942 \\
\hline $\operatorname{NEU}\left(\times 10^{9} / \mathrm{L}\right)$ & $0.9(0.8-0.9)$ & $0.9(0.8-0.9)$ & $0.8(0.8-0.9)$ & 0.279 \\
\hline PLT $\left(\times 10^{9} / \mathrm{L}\right)$ & $171.0(104.0-275.0)$ & $162.0(106.0-285.0)$ & $184.0(98.0-267.0)$ & 0.773 \\
\hline ALB $(g / L)$ & $32.0 \pm 3.9$ & $31.5 \pm 3.9$ & $32.4 \pm 4.0$ & 0.356 \\
\hline Mechanical ventilation (\%) & $37(60.7)$ & $20(66.7)$ & $17(54.8)$ & 0.344 \\
\hline ICU (days) & $6.0(4.0-10.0)$ & $5.0(4.0-7.0)$ & $7.0(5.0-14.0)$ & 0.009 \\
\hline
\end{tabular}

ALB, albumin; BMI, body mass index; CVP, central venous pressure; HB, hemoglobin; ICU, intensive care unit; MAP, mean arterial pressure; NEU, neutrophil; SOFA, sequential organ failure score; PLT, platelet; WBC, white blood cells.

Table 2 Comparison of puncture effects between the 2 groups

\begin{tabular}{lcccc}
\hline Variables & Total & Experimental group & Control group & P value \\
\hline Cases, $\mathrm{n}(\%)$ & 61 & $30(49.2)$ & $31(50.8)$ & $6.0(5.0-7.5)$ \\
Operation time (min) & $5.0(4.0-6.8)$ & $4.0(3.0-5.0)$ & $6.0(4.0-5.0)$ & $<0.001$ \\
Distance $(\mathrm{cm})$ & $5.0(4.0-6.0)$ & $6.0(5.0-7.0)$ & $28(93.3)$ & $22(71.0)$ \\
Success rate, $\mathrm{n}(\%)$ & $50(82.0)$ & 0.001 & 0.023 \\
\hline
\end{tabular}

the puncture point to the midpoint of the clavicle in the experimental group was longer than that of the control group, and the difference was statistically significant (6.0 vs. $4.0 \mathrm{~cm}, \mathrm{P}<0.001)$. The total operating time of the experimental group was significantly shorter (4.0 vs. $6.0 \mathrm{~min}, \mathrm{P}<0.001)$, and the difference was statistically significant (Table 2).

\section{Comparison of complications between the 2 groups}

The incidence of complications in the control group was significantly higher than that in the experimental group
( $45 \%$ vs. $12.5 \%)$, and the difference was statistically significant $\left(\chi^{2}=10.313, \mathrm{P}=0.001\right)$, as shown in Figure 3.

\section{Discussion}

For critically ill patients, intravenous infusion technology is an important technology for life support (1). In patients with septic shock, low blood volume, poor peripheral circulation, and other factors often lead to poor venous filling and severe edema of the limbs. After the peripheral blood vessels are repeatedly punctured, the operation is very difficult, and the fluid cannot be infused in time. Therefore, 


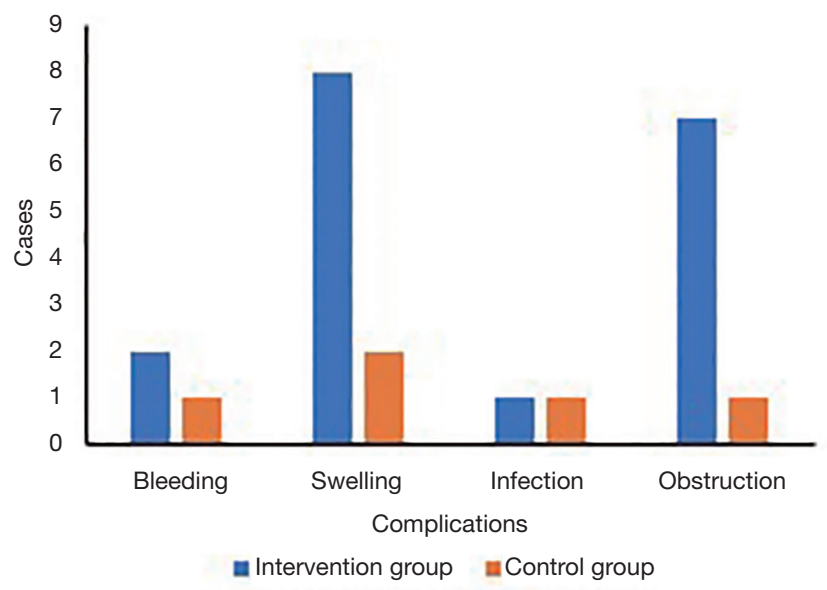

Figure 3 Comparison of complications between the 2 groups.

the choice of vein directly affects the success rate of puncture and has a greater relationship with the occurrence of complications, such as phlebitis (4). In the present study, we found that ultrasound-guided external jugular vein puncture can significantly increase the 1-time success rate and shorten the time required.

The external jugular vein is the largest branch of the superficial cervical vein. It is formed by the confluence of the posterior branch of the posterior mandibular vein and the posterior auricular vein near the mandibular angle (5). There are no important accompanying nerves and aorta around it. The vein has a large lumen and superficial filling. With abundant blood flow, irritant and hypertonic drugs can be rapidly diluted to avoid drug stimulation and damage to the blood vessel wall. Because the external jugular vein is closer to the heart than the limb veins, the drug can quickly flow back to the heart and be delivered to all parts of the body. Therefore, treatment with external jugular vein infusion can significantly improve the rescue success rate $(5,6)$. Studies have shown that because the external jugular vein is full and straight, it can effectively reduce the incidence of needle prolapse $(7,8)$, resulting in less frequent swelling of blood vessels, and the indwelling time of the vein can reach 4-7 days. Therefore, for patients with septic shock without deep venous catheterization, external jugular vein catheterization is the first and most effective treatment option. However, due to the short exposure length of the external jugular vein on the body surface, repeated puncture is not suitable, and requires nurses to increase the success rate of 1 puncture. It has been reported that different puncture methods will affect the success rate of the first puncture (9). In the present study, we found that when the diameter of the external jugular vein is measured using an ultrasound instrument, the average diameter is between $0.25 \pm 0.05 \mathrm{~cm}$, and the diameter of a common clinical trocar is $0.18-0.2 \mathrm{~cm}$; this can greatly improve the success rate of the first puncture under direct vision. The success rate of blind puncture mainly depends on the patient's blood vessel condition and the experience of the operator. The probability of the first puncture is relatively small, and the puncture effect is very uncertain. The use of ultrasound to measure the diameter of the vein and the placement of a peripheral venous indwelling needle guided by ultrasound has the advantages of accuracy, speed, safety, and high success rate of the first puncture (10).

In the present study, we found that, compared with traditional blind puncture with bare hands, ultrasoundguided external jugular vein puncture and catheterization has the following advantages. First, the external jugular vein can be accurately positioned, and the shape and diameter of the blood vessel can be clearly explored. The operator can use the help of the "perspective" effect of ultrasound, and operations, such as puncture and tube placement, are accurately completed (11), which greatly improves the success rate of a puncture and reduces the harm of repeated punctures. At the same time, ultrasound-guided puncture shortens the operation time. Second, traditional blind puncture may cause repuncture after needle insertion due to poor control of the needle-insertion angle and depth. After repeated repuncture, it may cause exudation around the blood vessel. Ultrasound guidance can clearly display the needle tip position and observe the needle depth in real time, thereby reducing the blood vessel wall and soft tissue damage, as well as fluid extravasation, phlebitis, and other complications. The distance between the puncture point and the clavicle midpoint during ultrasound-guided external jugular vein puncture determines the number of punctures. For example, if a puncture is unsuccessful, the probability of successful puncture again is greatly reduced the closer it is to the clavicle. Therefore, the longer the distance between the puncture point and the clavicle midpoint in the experimental group is, the more beneficial it is to use the vein, which conforms to the principle of using the vein from the distal end to the proximal end.

In clinical nursing work, the problem of difficult venipuncture is often encountered. For blood vessels that are poorly visible to the naked eye, nurses can apply this new technology to solve the problem, which can not only effectively increase the success rate of a puncture but can 
reduce puncture complications It also greatly reduces the discomfort of patients, doctor-patient disputes, and workload. However, the operator's proficiency in the use of ultrasound is also an important factor in determining the puncture time and the incidence of complications (12). In the clinical setting, a number of steps must be followed. First, the principle of aseptic operation must be strictly followed. Compared with blind puncture, ultrasoundguided venipuncture adds probes. Although there are sterile protective sleeves and sterile coupling agents, the chance of infection increases; therefore, this requires operators to have a strict concept of sterility to prevent infection (13). Second, the probe needs to be vertical to the skin, and the action should be gentle during operation. Excessive force should be avoided to compress the deflated blood vessels to affect the image quality. The hand holding the probe should find a suitable fulcrum to ensure that the blood vessel image is placed in the center of the display (14). Because the needle tip is not covered by the cannula and the metal structure is the strongest ultrasonic echo, the speed of the needle should be slowed down after entering the upper wall of the blood vessel to ensure that the needle tip can be clearly imaged. If the high echo highlight is found to be offset, the direction and angle should be adjusted in time. Third, the angle should be $15-30^{\circ}$ when inserting the needle. The speed should be fast and then slow. After the needle tip is developed, the needle should be leveled before pushing the cannula (15). Fourth, when the patient's blood volume is low and peripheral circulation is poor, ultrasound shows that the needle tip has entered the blood vessel, but there is still no blood return in the cannula. At this time, it is uncertain whether the cannula is in the blood vessel. Therefore, a syringe should be used for suction judgment. In 5 of our cases in experimental group, the catheter was successfully placed, but no blood returned. Aspiration was required to confirm that the catheter was successfully placed.

\section{Conclusions}

For patients in the ICU with sepsis, especially when they have hypotension, poor peripheral circulation, and the external jugular vein cannot be identified by the naked eye, ultrasound-guided external jugular vein puncture can greatly increase the success rate of a puncture and reduce complications. In our opinion, this method is worthy regularly applied in ICU practice, especially for patients with sepsis. The sample size of this study was relatively small and further study involved more patients should be warranted.

\section{Acknowledgments}

Funding: Chinese PLA General Hospital Clinical Research Supportive Fund (No. 2018FC-WJFWZX-2-14).

\section{Footnote}

Reporting Checklist: The authors have completed the CONSORT reporting checklist. Available at http://dx.doi. org/10.21037/apm-20-2500

Data Sharing Statement: Available at http://dx.doi. org/10.21037/apm-20-2500

Conflicts of Interest: All authors have completed the ICMJE uniform disclosure form (available at http://dx.doi. org/10.21037/apm-20-2500). The authors have no conflicts of interest to declare.

Ethical Statement: The authors are accountable for all aspects of the work in ensuring that questions related to the accuracy or integrity of any part of the work are appropriately investigated and resolved. The present study was approved by the Ethics Committee of the First Medical Center of the PLA General Hospital (approval number: 20200328), and written informed consent was provided by the patient or family members. All procedures performed in this study involving human participants were in accordance with the Declaration of Helsinki (as revised in 2013).

Open Access Statement: This is an Open Access article distributed in accordance with the Creative Commons Attribution-NonCommercial-NoDerivs 4.0 International License (CC BY-NC-ND 4.0), which permits the noncommercial replication and distribution of the article with the strict proviso that no changes or edits are made and the original work is properly cited (including links to both the formal publication through the relevant DOI and the license). See: https://creativecommons.org/licenses/by-nc-nd/4.0/.

\section{References}

1. Schmidt GA, Blaivas M, Conrad SA, et al. Ultrasoundguided vascular access in critical illness. Intensive Care Med 2019;45:434-46. 
2. Stolz La, Stolzu, Howe C, et al. Ultrasound-guided peripheral venous access: a meta-analysis and systematic review. J Vascular Access 2015;16:321-6.

3. Singer M, Deutschman CS, Seymour CW, et al. The Third International Consensus Definitions for Sepsis and Septic Shock (Sepsis-3). JAMA 2016;315:801-10.

4. Scales K. Vascular access: a guide to peripheral venous cannulation. Nurs Stand 2005;19:48-52.

5. Mitre CI, Golea A, Acalovschi I, et al. Ultrasound-guided external jugular vein cannulation for central venous access by inexperienced trainees. Eur J Anaesthesiol 2010;27:300-3.

6. Leprêtre P, Clavier T, Ménard AL, et al. Rescue stem cell allograft in intensive care unit patients during septic shock with multi-organ failure. J Crit Care 2019;54:122-4.

7. Mallet F, Perret M, Tran T, et al. REALISM group. Early herpes and TTV DNAemia in septic shock patients: a pilot study. Intensive Care Med Exp 2019;7:28.

8. Kato K, Taniguchi M, Iwasaki Y, et al. Central venous access via external jugular vein with CT-venography using a multidetector helical 16-section CT. J Invest Surg 2014;27:176-82.

9. Kim TE, Howard SK, Funck N, et al. A randomized comparison of long-axis and short-axis Imaging for inplane ultrasound-guided popliteal-sciatic perineural

Cite this article as: Luo S, Xue Y, Tang S, Hou S, Pei M. Application of ultrasound-guided external jugular vein puncture in intensive care unit (ICU) patients with severe sepsis: a randomised trial. Ann Palliat Med 2021;10(1):530-537. doi: 10.21037/apm-20-2500 catheter insertion. J Anesth 2014;28:854-60.

10. Duffy BL, Lee JS. Intravenous access: a comparison of two methods. Anaesth Intensive Care 1983;11:135-7.

11. Napoli M, Montinaro A, Russo F, et al. Early experiences of intraoperative ultrasound guided angioplasty of the arterial stenosis during upper limb arteriovenous fistula creation. J Vasc Access 2007;8:97-102.

12. Liu Y, Liu M, Han D, et al. Potential of modified puncture method to decrease intravenous indwelling needle-related complications in inpatients with cardiovascular disease. J Int Med Res 2019;47:3133-9.

13. Li Z, Chen L. Comparison of ultrasound-guided modified Seldinger technique versus blind puncture for peripherally inserted central catheter: a meta-analysis of randomized controlled trials. Crit Care 2015;19:64.

14. Komolafe O, Olatise O. Utility of blind percutaneous jugular venous cannulation in resource-limited settings. J Vasc Access 2017;1:26-29.

15. Jiang M, Mao JL, He B. Clinical definition of the axillary vein and experience with blind axillary puncture. Int J Cardiol 2012;159:243-5.

(English Language Editor: R. Scott) 